\title{
Dimensional accuracy of ankle-foot orthoses constructed by rapid customization and manufacturing framework
}

\author{
Elisa S. Schrank, BS; ${ }^{1}$ Steven J. Stanhope, $\mathbf{P h D}^{1-2 *}$ \\ ${ }^{1}$ Department of Mechanical Engineering, ${ }^{2}$ Department of Health, Nutrition and Exercise Sciences, and Biomechanics \\ and Movement Science Interdisciplinary Program, University of Delaware, Newark, DE
}

\begin{abstract}
Passive-dynamic ankle-foot orthoses (PD-AFOs) constitute a class of ankle braces that rely on material properties and physical features to establish functional characteristics such as bending or rotational stiffness. We have developed a novel framework that combines a fully parameterized PD-AFO computer-aided design (CAD) model and free-form fabrication to rapidly manufacture customized PD-AFOs. The threedimensional locations of select anatomic landmarks serve to fit customize the PD-AFO CAD model. A virtual orthopedic alignment process and selection of discrete design parameter values further customize the orthosis, which is fabricated via selective laser sintering. CAD models were customized and fullscale orthoses were manufactured for two nondisabled subjects. The surface of one half-scale CAD model was marked with $3 \mathrm{~mm}$ hemispherical dimples, and four orthoses were manufactured in different build orientations and positions. Dimensional accuracy was determined by calculating discrepancies between corresponding $\mathrm{CAD}$ and fabricated orthoses interdimple distances. Subjective evaluations of the full-scale PD-AFOs following use in gait were positive. Dimension discrepancies were well under a $2 \mathrm{~mm}$ tolerance for the four half-scale orthoses. Mean foot plate, strut, and cuff component discrepancies were $0.31+/-$ $0.28,0.34+/-0.08,0.52+/-0.39 \mathrm{~mm}$, respectively, and $0.29+/-$ $0.23 \mathrm{~mm}$ for the overall orthosis. Dimensional accuracy of the rapid customization and manufacturing framework was well within tolerances suggested in the literature.
\end{abstract}

Key words: ankle, computer-aided design, customization, gait, orthosis, orthotics, parameterization, rehabilitation, selective laser sintering, three-dimensional printing.

\section{INTRODUCTION}

According to the Americans with Disabilities report, in 2005 approximately 27 million people over the age of 15 had a walking-related disability [1]. Ankle joint musculature is extremely important during walking and is thought to be the primary muscle group that supports upright stance and produces forward propulsion [2-3]. Individuals with muscular weakness about the ankle, an impairment often caused by upper motor neuron disorders and lower-limb injuries, are frequently prescribed ankle-foot orthoses (AFOs), which brace the ankle during gait and aim to improve gait function [4].

Passive-dynamic AFOs (PD-AFOs) constitute a class of ankle braces that rely on material properties and physical features to establish functional characteristics such as bending or rotational stiffness and the storage and return of mechanical energy [5]. PD-AFOs are traditionally composed of foot plate, strut, and cuff components, which may

Abbreviations: $2-\mathrm{D}=$ two-dimensional, 3 - $\mathrm{D}=$ three-dimensional, AFO = ankle-foot orthosis, CAD = computer-aided design, $\mathrm{PD}-\mathrm{AFO}=$ passive-dynamic AFO, SD = standard deviation, SLS = selective laser sintering.

*Address all correspondence to Steven J. Stanhope, PhD; 211 McDowell Hall, University of Delaware, Newark, DE 19716-5710; 302-831-3496; fax: 302-831-3490.

Email: stanhope@udel.edu

DOI:10.1682/JRRD.2009.12.0195 
be fabricated using continuous material [5-7] or connected as components in various manners [8-9]. Despite the great potential for biomechanical assessment and treatment using this classification of orthoses, currently prescribed PDAFOs are often generic, having standardized size and shape (fit) and bending or rotational stiffness (functional) characteristics [10-11]. When customization of functional characteristics is sought, PD-AFOs are typically manually fabricated by orthotists - which may introduce undesired manufacturing variability in PD-AFO quality or effectiveness, depending on an orthotists' skill and experience [12] — and require substantial time and expertise to ultimately manufacture orthoses having functional characteristics that match the unique gait dynamics of each patient [6-7,13]. Furthermore, the annual cost of these devices is substantial. In 2007, the prescription of orthotics accounted for US $\$ 458,000,000$ of Medicare expenditures alone [14]. Therefore, the capability to rapidly design and manufacture customized orthoses with precisely controlled characteristics would help transform the PD-AFO customization and fabrication process from a craft-based industry into a modern clinical specialty [15].

Fit customization is an important design factor for obtaining optimal function from a PD-AFO. The size and shape characteristics, which describe the fit of a PDAFO, can be customized through a variety of methods. Traditionally, an orthotist casts a patient's shank and foot to create a negative mold. A positive mold is generated from the negative mold, and then the PD-AFO is manually fabricated around this positive mold similar to methods for fabricating a foot orthosis [16]. While manual manufacturing methods can sufficiently generate a PDAFO with customized size, augmented shape, and functional characteristics, manual manufacturing methods can contribute to undesirable variability in quality of manufactured components.

Recent efforts have worked to utilize computer-aided design (CAD) models and associated parameterization tools to customize orthoses. Darling and Sun designed a parameterized orthosis model that consisted of two rigid components, one for each the foot and shank, which were attached by a single-degree-of-freedom hinge [17]. Parameterization of this model was based on two anatomically relevant coordinate systems, one for each of the components. Patient-specific imaging data were fit to the parameterized model to scale the orthosis. While this CAD model was parameterized for size and ankle angle, the orthosis design lacked the organic shape characteristics and parameterization of orthosis functional characteristics.
In this article, we define parameterization as the process of identifying the parameters necessary for the complete specification as well as manipulation of the PDAFO CAD model. Using parameters to specify and manipulate a PD-AFO CAD model has profound implications for the objective and precise customization of biomechanically designed orthoses that match patient needs with orthosis function [15]. For example, the overall size of a CAD model can be readily and objectively increased by a discrete percentage when the general scale parameter value is changed. By forming interparameter hierarchal dependencies, single parameter values can control more complex PD-AFO characteristics (e.g., the radial expansion of a cuff's inner surface to accommodate padding thickness). Tuned functional characteristics of a PD-AFO such as rotational or bending stiffness may be readily achieved when a hierarchy of shape-related parameters is optimized to generate a CAD model having optimal component size, shape, and thickness. While the full parameterization of a CAD model provides a powerful means for designing PD-AFOs with unique and highly personalized characteristics, manufacturing these PD-AFOs requires techniques beyond the traditional PD-AFO manufacturing practices.

Rapid free-form manufacturing techniques, such as selective laser sintering (SLS), allow for the rapid fabrication of unique parts. SLS is an additive process by which a carbon dioxide laser beam selectively heats and fuses (sinters) the powder material into a solid part. Layer by layer, the powder is evenly rolled out over the machine bed, and the laser draws a cross section of the part into each subsequent powder layer. The surrounding unsintered material provides the support structure for the part. An advantage of the SLS process is that cost of manufacturing is primarily a function of part volume and not part intricacy, thus this method is ideal for fabricating objects with unique, complex geometry. Recently, the use of SLS for fabrication of lower-limb prosthetic sockets [18-19], dynamic prosthetic feet [20], and the mass customization of foot orthoses [21] has been investigated. Faustini et al. demonstrated the feasibility of using SLS and manual CAD procedures to duplicate the functional characteristics of carbon composite PD-AFOs [5], while South et al. demonstrated this process using prosthetic feet [20].

While these recent studies have demonstrated the feasibility of using SLS manufacturing techniques for dynamic prosthetic and orthotic applications, previous reports indicate that dimensional accuracy of SLS-fabricated parts can 
vary because of several factors, including part shrinkage and SLS build parameters [22-25]. This drawback is a concern when parts are manufactured that require specific dimensions, such as the size and shape of the force-bearing surface of the cuff and foot outline of a PD-AFO. Building a scale part to determine the necessary scale factors for a particular material type and set of build parameters is a standard technique used to improve dimensional accuracy of SLS fabricated parts. However, the scale part does not account for part-specific factors such as build orientation and position and geometry-dependent shrinkage [24]. Thus, the dimensional accuracy must be assessed on a part-bypart basis.

We have developed a PD-AFO automated customization and manufacturing framework that supports the automated fit and functional customization, SLS manufacturing, and subsequent assessment of PD-AFOs. A submethod of the framework, which relates to fit customization, contains novel subject fit characterization and orthopedic alignment techniques. The framework harnesses the strengths of a fully parameterized PD-AFO CAD model and SLS manufacturing to rapidly customize and fabricate PD-AFOs. The purpose of this study was to objectively assess the overall dimensional accuracy while subjectively assessing fit of PD-AFOs resulting from the fit customization and manufacturing framework.

\section{METHODS}

\section{Overview of Fit Customization and Manufacturing Framework}

The automated customization and manufacturing framework consists of five primary stages (Figure 1). While the overall framework contains both fit and function customization, the focus of this article relates to the steps that primarily influence size and shape of the manufactured device. During stage 1, the size and shape characteristics needed for the orthosis are digitally captured from the subject as discrete three-dimensional (3-D) digitized landmarks. These digitized landmarks consist of segment defining as well as shape characterization landmarks. Next, the segment landmarks are used to form joint centers for the knee and ankle and anatomically relevant segment coordinate systems for the shank and foot. Shape characterization landmarks are then registered to the segment

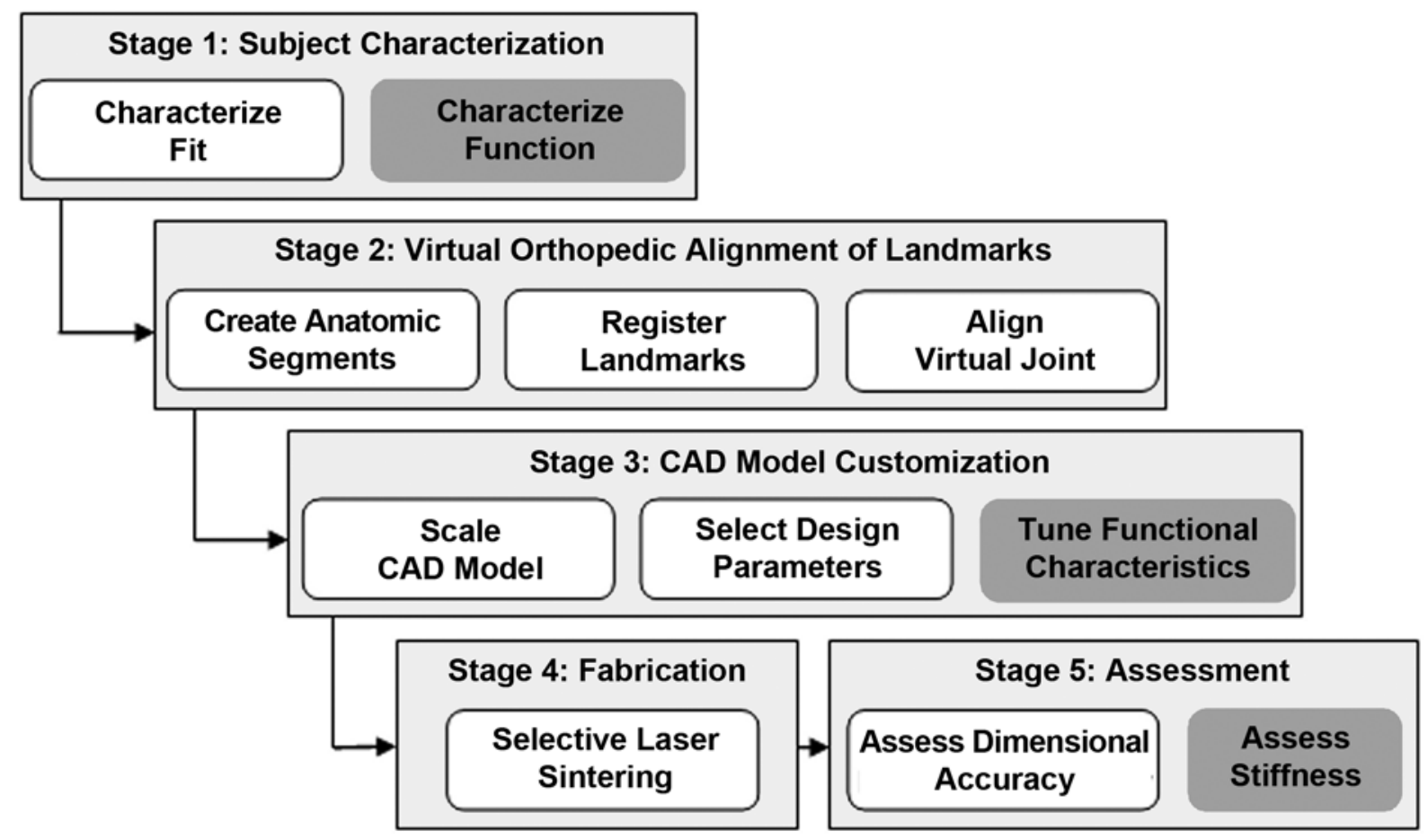

Figure 1.

Schematic outlining five sequential stages of orthosis customization and manufacturing framework. Grayed-out boxes indicate steps not associated with fit customization and manufacturing subframework and therefore not addressed in this article. CAD = computer-aided design. 
coordinate systems. All landmarks then undergo virtual orthopedic alignment by rotation of the shank coordinate system and related landmarks relative to the foot segment about a shared virtual ankle joint center. The virtual orthopedic alignment process produces landmark location data that serve as the custom fit parameters for the fully parameterized CAD model. Select design parameters may then be employed to further customize the CAD model. The customized CAD model is then rapidly fabricated via SLS and assessed for dimensional accuracy.

\section{CAD Model}

The fully parameterized PD-AFO CAD model was constructed in Inventor Professional v11 (Autodesk, Inc; San Rafael, California). The CAD model contains three primary components (foot plate, strut, and cuff) joined by upper and lower spans (Figure 2). The foot plate two-dimensional (2-D) profile is characterized by the planar locations of

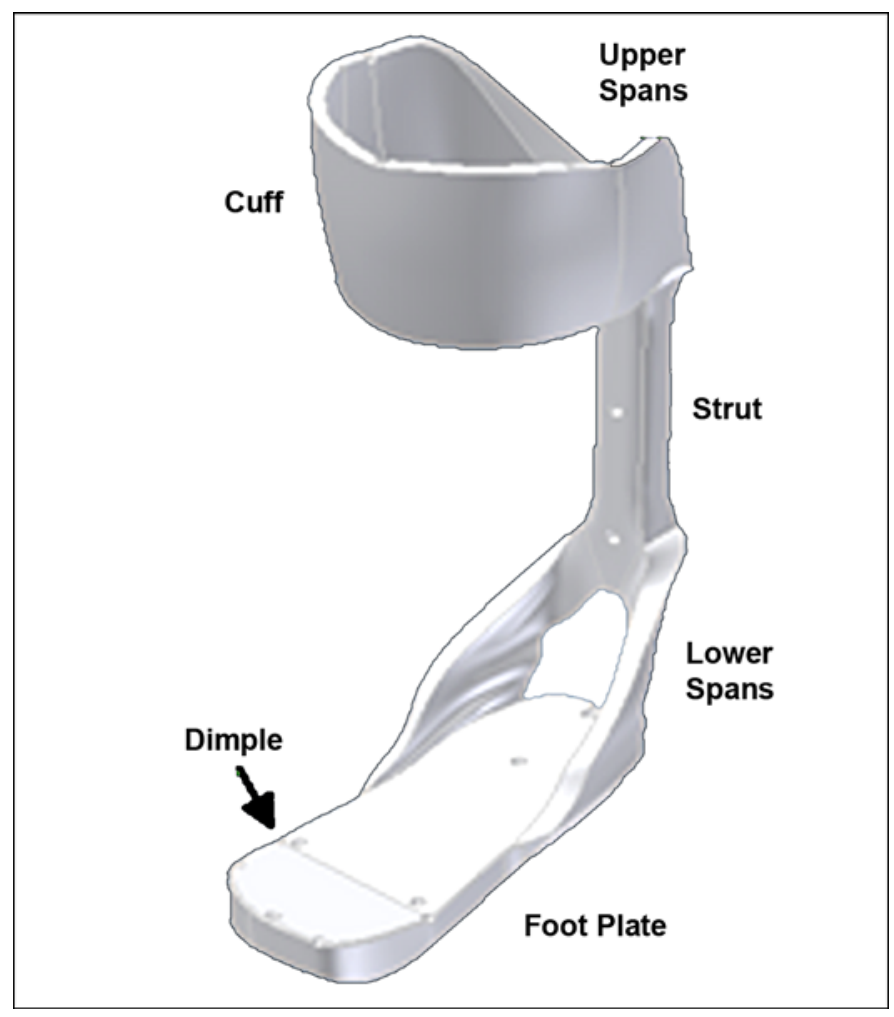

Figure 2.

Passive-dynamic ankle-foot orthosis fit customization and manufacture framework: right-side orthosis during computer-aided design model customization stage. Cuff, posterior strut, foot plate components, and upper and lower spans are labeled. Location of one of the $3 \mathrm{~mm}$ dimples used for dimensional accuracy measures is indicated.
10 foot shape characterization landmarks (Figure 3) connected by a series of splines.

The strut component is sized as a function of shank length and shaped by a series of 11 parallel, 2-D profiles that are offset in the posterior direction from the shank segment longitudinal axis by an operator-specified distance. The 11 profiles are lofted together to form a 3-D strut component. The top and bottom strut segments are beveled to enable attachment of the upper and lower spans.

The cuff component is automatically sized, shaped, and positioned with four rows of lofted 3-D splines, each connecting a row of shank shape characterization landmarks (Figure 4(a)). The CAD model lofts together the splines to create an initial interior cuff surface (Figure 4(b)). The CAD model uses single parameter values to radially offset the cuff surface from the shank segment longitudinal axis to accommodate a desired padding thickness (Figure 4(c)) and to uniformly thicken the radially expanded cuff surface to create a custom 3-D cuff component (Figure 4(d)).

Once constructed, the foot plate, strut, and cuff are automatically connected by a set of lofted upper and lower spans, which are fit-controlled by the foot plate, strut, and cuff locations, as well as the digitized landmarks. The

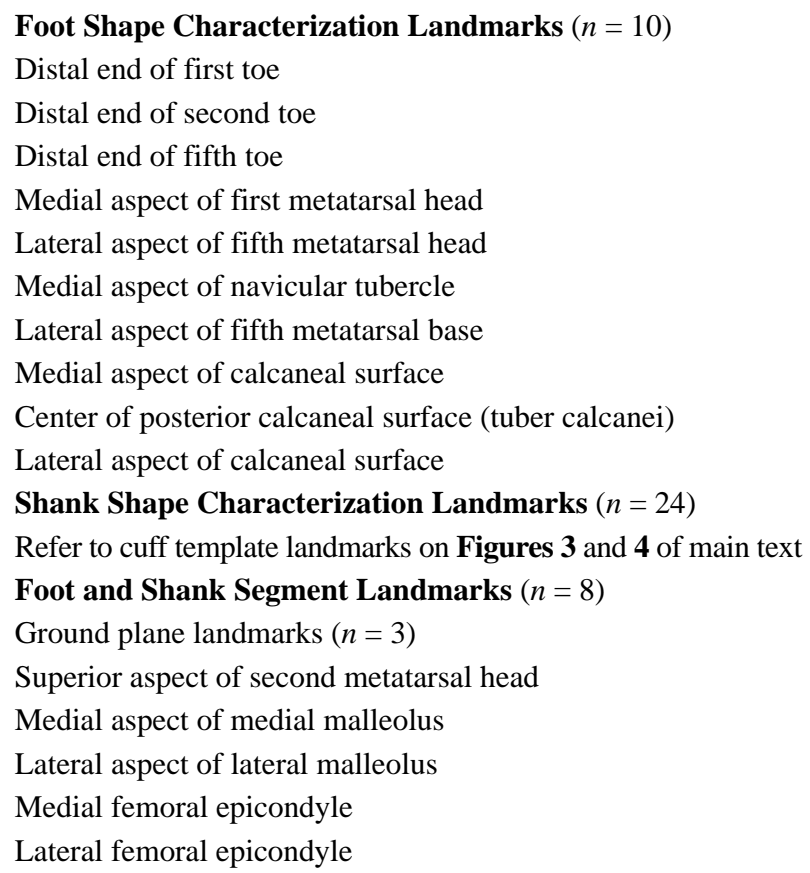

Figure 3.

Digitized three-dimensional landmarks acquired during subject fit characterization stage. 
inherent set of assembly rules assures a smooth connection between components, as well as clearance of the spans from the subject's bony prominences.

Lastly, additional design parameters were implemented to further control the fit of the PD-AFO CAD model. These design parameters included foot plate thickness, foot plate padding offset, cuff thickness, cuff padding offset, strut offset, and several additional foot plate shape parameters. The foot plate and cuff thickness parameters control the foot plate profile extrusion distance and interior cuff surface thickening distance, respectively. Because all design parameter values may be set by the operator and standardized before the subject-specific landmark data are loaded, the entire PD-AFO CAD model is constructed without operator involvement. For the purpose of this study, we added $3 \mathrm{~mm}$ hemispherical dimples to the CAD model foot plate, strut, and cuff (Figure 2) to enable subsequent dimensional accuracy assessment of each component (intracomponent) and the overall orthosis (intercomponent). (a)

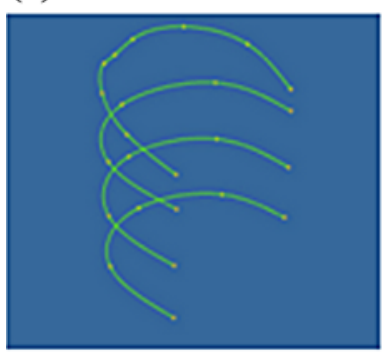

(d)
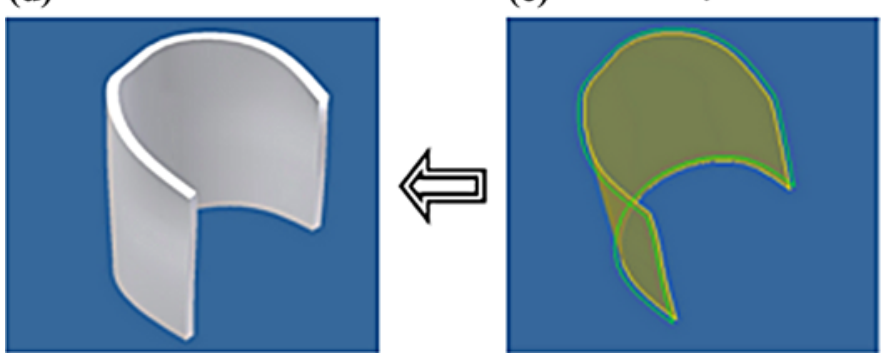

(c)

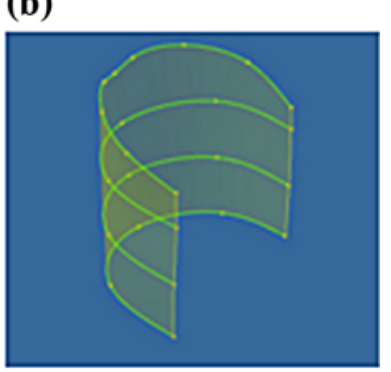

Figure 4.

Passive-dynamic ankle-foot orthosis computer-aided design model cuff construction process: (a) Four three-dimensional (3-D) splines connect four rows of shank shape characterization landmarks residing on cuff template. (b) Cuff surface is created via vertical lofting of four initial splines. (c) Cuff inner surface is radially offset from shank longitudinal axis to accommodate padding thickness. (d) Cuff surface is thickened outward by specified amount to form final 3-D cuff component.

\section{Subject Fit Characterization}

Following the receipt of informed written consent, 3-D digitized landmark data were obtained from the right lower limbs of two subjects: one nondisabled male subject (age 48 years, height $1.77 \mathrm{~m}$, mass $71.8 \mathrm{~kg}$ ) and one nondisabled female subject (age 21 years, height $1.65 \mathrm{~m}$, mass $59.9 \mathrm{~kg}$ ). We used these data to customize a fully parameterized PD-AFO CAD model and subsequently manufacture an orthosis for each subject. The subjects were positioned standing symmetrically on a pelvic stabilization stand wearing a white knee-high stocking (Figure 5). The pelvic stabilization stand helped the subject remain motionless during

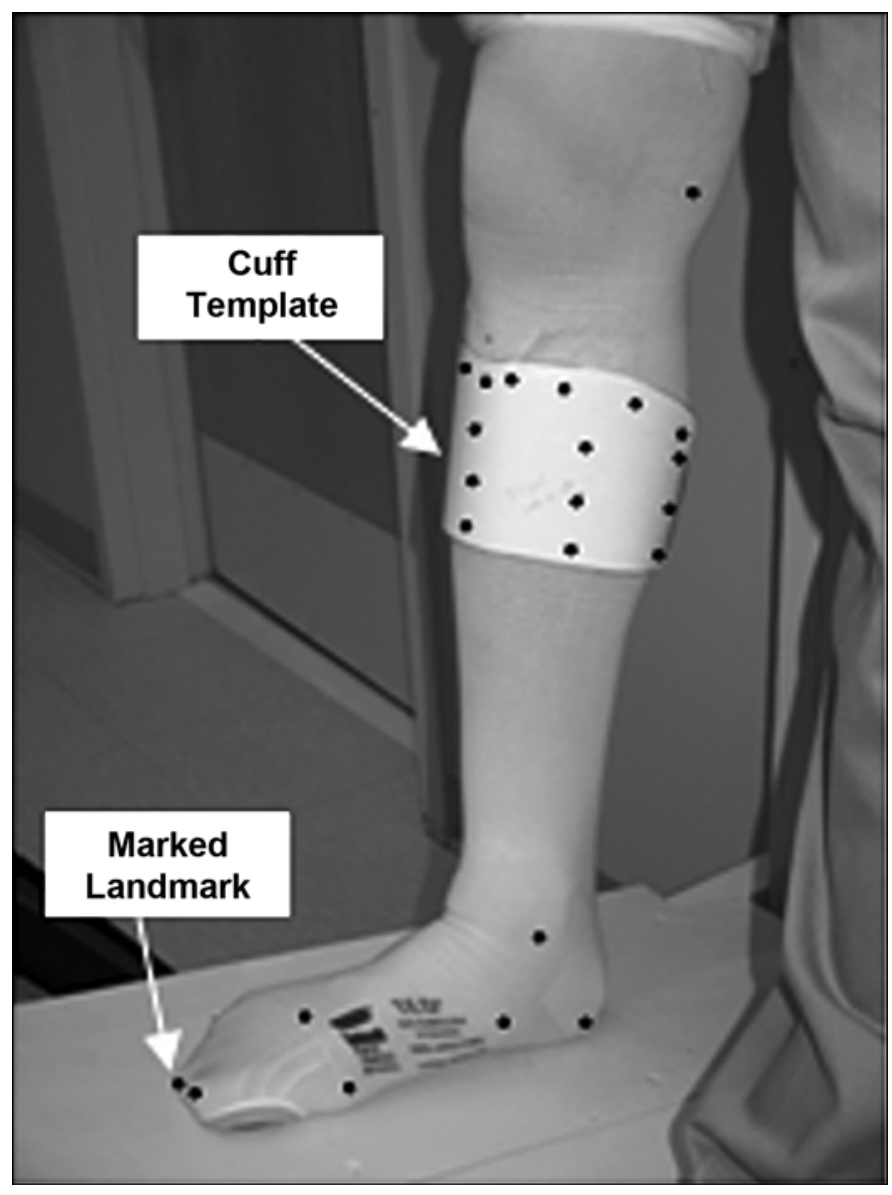

Figure 5.

Passive-dynamic ankle-foot orthosis fit customization and manufacture framework: medial side of subject's right leg during subject fit characterization stage, in which locations of marked landmarks are acquired using three-dimensional coordinate digitizer. Cuff template is sized, printed, positioned, and adhered to subject's leg by clinician. Note that size of marked landmark locations were enhanced for display purposes only. 
the digitization of anatomical landmarks. The stand consisted of a raised, backwardly inclined platform with attached backrest, on which subjects stood motionless by resting the posterior aspect of their pelvis against the backrest. Landmarks for characterizing foot shape as well as foot and shank segments, described in the Figure 3, were identified by palpation and marked. A customized outline of foot shape was obtained from a series of splines fit to the $10 \mathrm{foot}$ shape characterization landmarks. The three ground plane landmarks defined the standing support surface. The remaining foot and shank segment landmarks were used to locate joint centers and anatomically relevant and biomechanically aligned segment coordinate systems for the shank and foot segments. A cuff template was used to indicate the locations of 24 shank shape characterization landmarks. The cuff template was created in Inventor Professional v11 and printed on standard office paper. While the fully parameterized PD-AFO model accepts a range of cuff parameters, we scaled the height of the cuff template to 25 percent of the subject's shank length and width to 60 percent of the shank circumference to mimic customary PDAFO designs [6]. The template was positioned and secured to the subjects' leg by a clinician using adhesive tape (Figure 5). The subjects were instructed to remain still on the pelvic stabilization stand for approximately 1 minute while the 3-D location of each landmark was individually acquired with a calibrated 3-D Fusion FaroArm $( \pm 0.036 \mathrm{~mm}$ accuracy; FARO Technologies, Inc.; Lake Mary, Florida) and recorded in Geomagic Studio 9 (Geomagic, Inc; Research Triangle Park, North Carolina).

\section{Virtual Orthopedic Alignment}

The first step in the virtual orthopedic alignment process was establishing segment coordinate systems for the shank and foot. This process began with computing the locations of three virtual landmarks: the ankle joint center, knee joint center, and ankle joint center projection onto the ground plane. The ankle joint center and knee joint center locations were calculated as the midpoint of the line connecting the digitized medial and lateral malleoli and femoral epicondyle landmarks, respectively. We determined the location of the ankle joint center projection by translating the ankle joint center landmark to the ground plane along a displacement vector perpendicular to the ground plane. A vector connecting the ankle and knee joints formed the shank coordinate system longitudinal axis. The shank medial/lateral axis passed through the ankle joint center and resided in the segment's frontal plane, which passed through the ankle joint center and the digitized medial and lateral femoral epicondyle landmarks. The third axis of the shank coordinate system was orthogonal to the longitudinal and medial/lateral axes. The foot longitudinal axis was parallel to the ground plane and passed through the ankle joint center and the vertical projection of the digitized second metatarsal head landmark. The foot coordinate system medial/lateral axis was parallel to the ground plane and perpendicular to the foot longitudinal axis. The vector from the ankle joint center to ankle joint center projection served as the third axis of the orthogonal foot coordinate system. The ankle joint center served as the origin of both segment coordinate systems.

Next, the digitized and virtual landmarks were computationally registered (located) in their respective shank and foot coordinate systems. Registering the digitized and virtual landmarks enabled the final step of the virtual orthopedic alignment process, alignment of the virtual ankle joint. Custom-written MATLAB (The MathWorks, Inc; Natick, Massachusetts) scripts were used for calculating the 3-D orientation of the shank coordinate system relative to the foot coordinate system. The digitized and virtual shank segment and shape characterization landmarks were then computationally rotated with the shank coordinate system about three orthogonal axes such that the PD-AFO cuff and foot plate components conformed to a neutral orthosis orientation in which the shank coordinate systems was orthogonal to the foot coordinate system. The fully parameterized PD-AFO CAD model was then customized with the resulting 3-D landmark locations and a series of preselected design parameter values.

\section{Fabrication}

Four half-scale PD-AFOs were fabricated with SLS. Each PD-AFO was built in a different combination of orientation (strut horizontal [C, D] or vertical [A, B]) relative to the SLS laser beam and position (right, left, front, or back [A, B, C, D]) relative to SLS build volume (Figure 6). Half-scale PD-AFOs were built so PD-AFOs could be fabricated in various orientations and positions in a single build, because the size of the build volume restricted multiple full-scale PD-AFOs from being fabricated in the same build. The PD-AFOs were marked with the orientation and position in which they were built. All four PD-AFOs were fabricated in a single build in a Vanguard HS SLS machine (3D Systems Corporation; Rock Hill, South Carolina) with stable temperature control using DuraForm EX Natural Plastic (3D Systems). Manufacturer-indicated build 


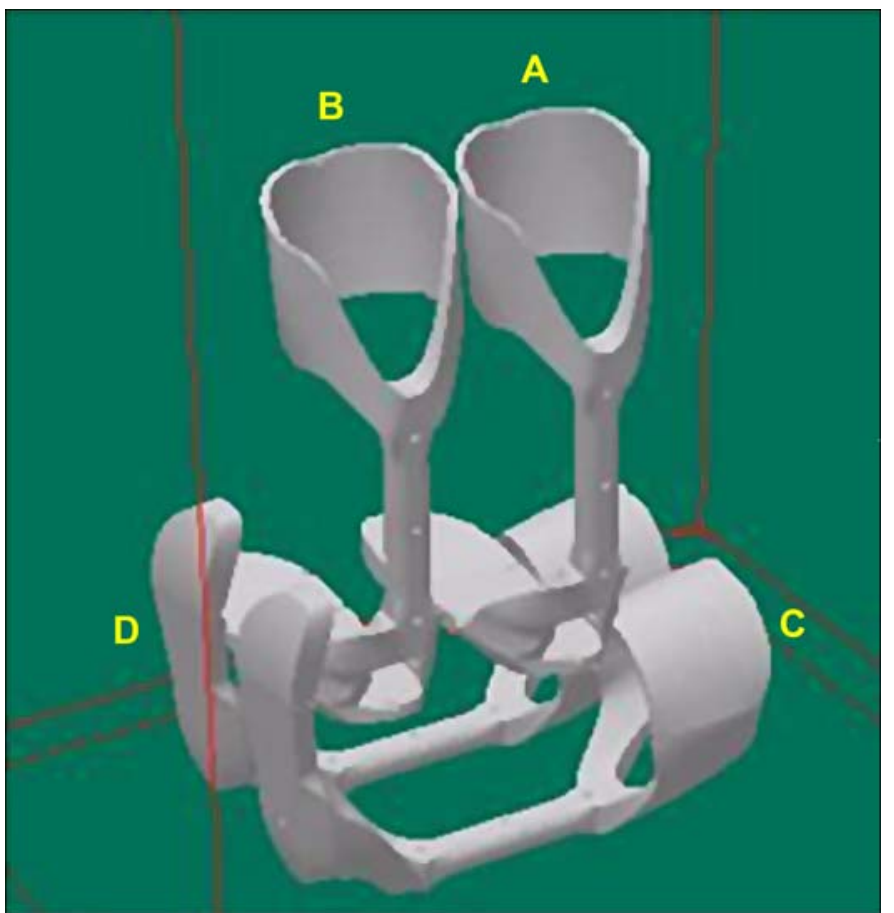

Figure 6.

Schematic indicating build orientations and positions of half-scale passive-dynamic ankle-foot orthoses within selective laser sintering build volume. Position $\mathrm{A}=$ right, $\mathrm{B}=$ left, $\mathrm{C}=$ front, and $\mathrm{D}=$ back.

settings were used, and scale factors were determined with the use of industry standard techniques to account for part shrinkage. In a separate build, two full-scale PD-AFOs were fabricated and stiffness tuned to 50 percent of the subjects' natural pseudo ankle stiffness so they could subjectively assess the fit customization during PD-AFO use [26].

\section{PD-AFO Assessment}

We measured 3-D interdimple distances (centroid to centroid) on the manufactured PD-AFOs using the FaroArm, fit with a $3 \mathrm{~mm}$ spherical tip. Each dimple was digitized three times for assessing repeatability. Three foot plate, one strut, and three cuff dimensions (3-D interdimple distance) were measured for assessing the intracomponent dimensional accuracy of each of the three components. Additionally, we measured three intercomponent dimensions, two between the foot plate and cuff and one between the foot plate and strut, to assess the dimensional accuracy of the overall orthosis. Discrepancies in experimental dimensions were computed relative to the corresponding CAD model dimension. The absolute maximum discrepancy, mean discrepancy, and the stan- dard deviation were determined for each dimension measurement. A dimension discrepancy tolerance of $2 \mathrm{~mm}$ was selected [27]. A two-way analysis of variance with repeated measures was performed to assess differences in the magnitude of discrepancies between constructed orthoses and corresponding CAD dimensions.

The full-scale PD-AFOs were padded with a thin, off-the-shelf, foam arch support secured on the foot plate surface and a $1 \mathrm{~cm}$-thick layer of foam padding adhered to the interior cuff surface. Straps were attached to the foot plate to help secure the subject's foot in the PDAFO. The subjects donned the padded PD-AFOs, and fit customization was visually assessed by a physical therapist. After the subjects walked in the PD-AFO for approximately 1 hour, their skin was examined for redness or signs of abrasion.

\section{RESULTS}

The 3-D distances for the intra- and intercomponent dimensions obtained from the PD-AFO CAD model ranged from 26.01 to $176.8 \mathrm{~mm}$ (Table). Prior to building, the SLS build volume was scaled with the use of industry standards by $1.0464,1.0469$, and 1.0350 percent in the SLS $x$-, $y$-, and $z$-build directions, respectively. The four half-scale PDAFOs were fabricated in less than 24 hours and extracted from the build volume following the customary cooldown period. Visual inspection of the fabricated PD-AFOs revealed no obvious manufacturing flaws (Figure 7).

The dimensional accuracy data were all within acceptable intra- and intercomponent dimensional accuracy tolerances-all dimension discrepancies were within the $2 \mathrm{~mm}$ limit. No dimension discrepancy was greater than $1.5 \mathrm{~mm}$, with the majority of the discrepancies below $0.5 \mathrm{~mm}$ (Figure 8). Across all PD-AFOs and all measured dimensions, the maximum absolute dimension discrepancies were 1.02, 0.42, 1.44 , and $0.88 \mathrm{~mm}$ for the foot plate, strut, cuff, and overall orthosis, respectively (Table). Overall mean discrepancies for the foot plate, strut, and cuff components were $0.31 \pm 0.28,0.34 \pm$ $0.08,0.52 \pm 0.39 \mathrm{~mm}$, respectively, and $0.29 \pm 0.23 \mathrm{~mm}$ for the overall orthosis. As can be seen from the data in the Table, good repeatability occurred in experimental measures across the three digitizing trials, as indicated by the low standard deviations (SDs) (maximum SD $=0.39$ $\mathrm{mm}$ ). No significant effects of the SLS build orientation and position on dimensional accuracy were found, yet a 
Table.

Absolute means ( $n=3) \pm$ standard deviations of seven intracomponent (foot plate, strut, cuff) and three intercomponent (foot plate cuff, foot plate strut) dimension discrepancies for each of four fabricated orthoses (A, B, C, D). Average means for each dimension discrepancy $(n=12)$ and orthosis $(n=30)$ are indicated in last row and last column, respectively. Grayed boxes contain dimensions obtained from CAD model.

\begin{tabular}{|c|c|c|c|c|c|c|c|c|c|c|c|}
\hline \multirow{3}{*}{ PD-AFO } & \multicolumn{10}{|c|}{ Dimension (mm) } & \multirow{3}{*}{ Average } \\
\hline & \multicolumn{3}{|c|}{ Foot Plate } & \multirow{2}{*}{$\begin{array}{c}\text { Strut } \\
4\end{array}$} & \multicolumn{3}{|c|}{ Cuff } & \multicolumn{2}{|c|}{ Foot Plate Cuff } & \multirow{2}{*}{$\begin{array}{c}\begin{array}{c}\text { Foot Plate } \\
\text { Strut }\end{array} \\
10\end{array}$} & \\
\hline & 1 & 2 & 3 & & 5 & 6 & 7 & 8 & 9 & & \\
\hline$\overline{\text { CAD Model }}$ & 26.01 & 42.83 & 118.23 & 66.58 & 44.76 & 55.95 & 42.42 & 176.80 & 134.37 & 83.06 & \\
\hline A & $0.13 \pm 0.10$ & $0.66 \pm 0.17$ & $0.41 \pm 0.07$ & $0.41 \pm 0.01$ & $0.09 \pm 0.08$ & $0.86 \pm 0.11$ & $0.61 \pm 0.22$ & $0.44 \pm 0.11$ & $0.28 \pm 0.25$ & $0.03 \pm 0.01$ & $0.39 \pm 0.28$ \\
\hline B & $0.11 \pm 0.07$ & $0.36 \pm 0.25$ & $0.34 \pm 0.18$ & $0.41 \pm 0.01$ & $0.14 \pm 0.12$ & $0.87 \pm 0.06$ & $0.56 \pm 0.12$ & $0.68 \pm 0.04$ & $0.57 \pm 0.31$ & $0.15 \pm 0.02$ & $0.42 \pm 0.27$ \\
\hline $\mathrm{D}$ & $0.10 \pm 0.06$ & $0.51 \pm 0.39$ & $0.18 \pm 0.07$ & $0.23 \pm 0.02$ & $0.27 \pm 0.16$ & $0.92 \pm 0.10$ & $0.14 \pm 0.05$ & $0.42 \pm 0.01$ & $0.38 \pm 0.18$ & $0.13 \pm 0.02$ & $0.33 \pm 0.27$ \\
\hline Average & $0.11 \pm 0.06$ & $0.58 \pm 0.29$ & $0.24 \pm 0.18$ & $0.34 \pm 0.08$ & $0.24 \pm 0.19$ & $0.97 \pm 0.20$ & $0.35 \pm 0.27$ & $0.42 \pm 0.21$ & $0.35 \pm 0.24$ & $0.11 \pm 0.06$ & - \\
\hline
\end{tabular}

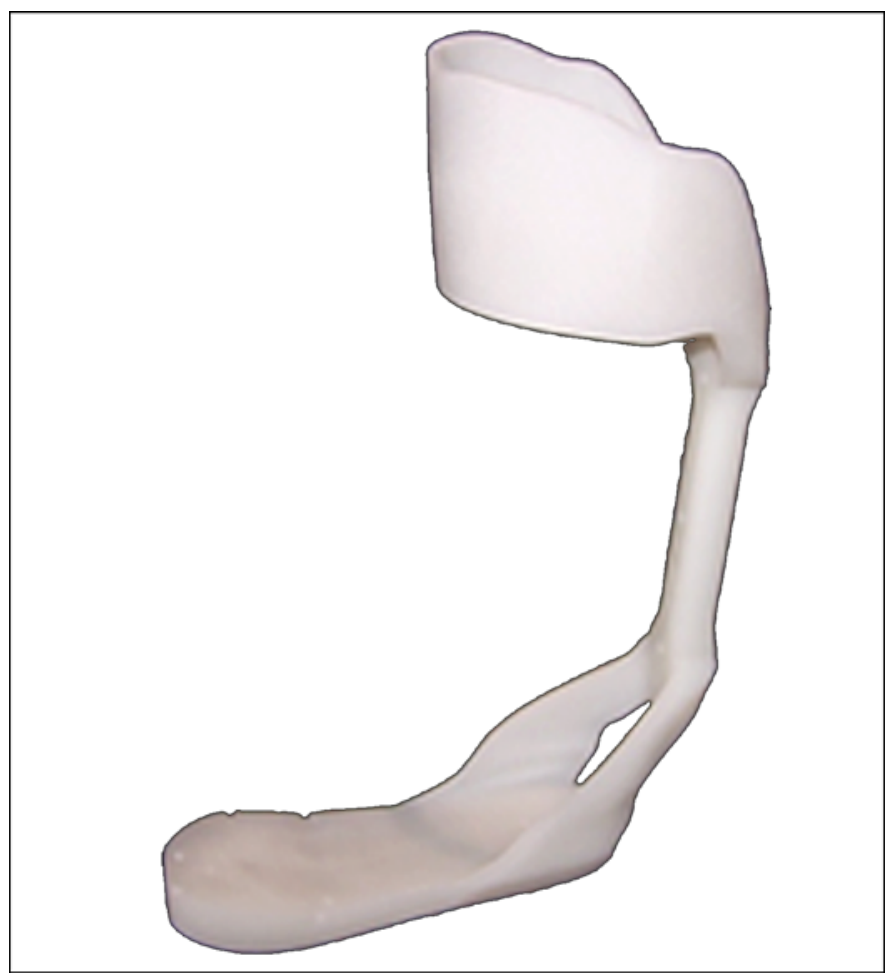

Figure 7.

Passive-dynamic ankle-foot orthosis (PD-AFO) fit customization and manufacture framework: medial aspect of subsequent PD-AFO fabricated using selective laser sintering.

significant effect was found of the dimension location on dimensional accuracy $\left(F_{3,9}=41.41, p<0.001\right)$. A significant interaction effect between the location of the dimension and the SLS build orientation and position was also found $\left(F_{3,9}=4.94, p<0.001\right)$.

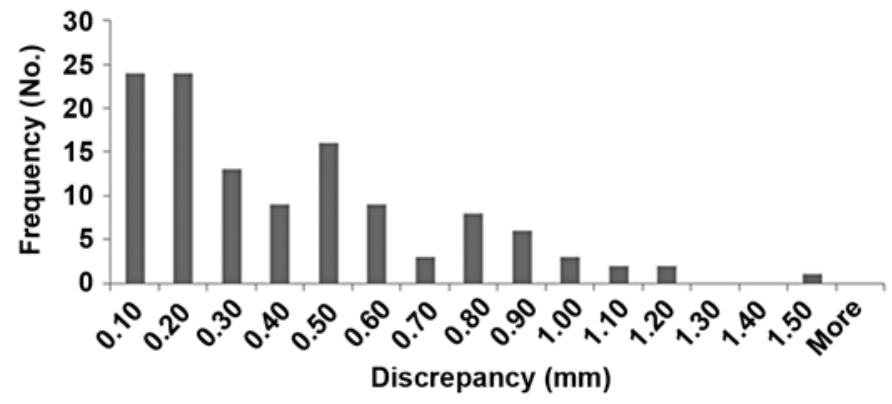

Figure 8.

Frequency distribution of absolute discrepancies (mm) between computer-aided design model and fabricated passive-dynamic anklefoot orthosis (PD-AFO) dimensions (corresponding three-dimensional interdimple distances). Individual data values (mm) from all four PDAFOs, including all 10 measured dimensions and all 3 digitizing trials, are displayed.

Following a period of walking in the full-scale orthosis for approximately 1 hour, the subjects demonstrated a fully accommodated, smooth, and rhythmic gait pattern and reported no discomfort. Upon visual inspection by a clinician, the skin under the cuff and about the foot showed no signs of uneven pressure distribution, redness, or abrasions for either subject.

\section{DISCUSSION}

This study aimed to evaluate the dimensional accuracy of PD-AFOs fabricated via our fit customization and manufacturing framework. With our method, the dimensional accuracy of the fabricated PD-AFOs was generally 
greater than that of other SLS-fabricated parts from previous studies, which reported mean absolute dimensional discrepancies between 0.89 and $1.0 \mathrm{~mm}$ [22-23,25]. These previous studies manufactured craniofacial models as opposed to PD-AFOs, and the difference in part geometry may explain the variation in results. Additionally, the craniofacial models were made based on reconstructed imaging data, and fabricated model dimensions were compared with the imaging scans. Therefore, the accuracy of the fabricated parts was based on the precision of reconstructing and obtaining dimension measurements from the imaging data as well as the accuracy of the SLS manufacturing process. In contrast, to assess dimensional accuracy, we compared fabricated PD-AFO dimensions to the CAD model dimensions, eliminating possible sources of error from the subject fit characterization and virtual orthopedic alignment stages. The fit customization assessment was used to further evaluate the accuracy of the fabricated PD-AFOs, because this step compared the fabricated PDAFO with the subjects, from whom the original digitized landmark data were collected.

Examination of the interaction effect between the location of the dimension and the SLS build orientation and position showed that the PD-AFOs built with the strut oriented horizontal to the SLS laser beam had lower dimensional discrepancies in strut height and cuff height (dimensions 4 and 7 in the Table) than PD-AFOs built with the strut oriented vertical to the laser beam. However, the cuff width (dimension 5 in the Table) was more accurate in PD-AFOs built with the strut vertical to the SLS laser beam as opposed to in PD-AFOs built with the strut horizontal to the laser beam. These data may suggest that although no overall significant effect of PD-AFO orientation and position was found, dimensional accuracy may improve when the longitudinal axis of a part is oriented horizontally, or perpendicular to the SLS laser beam. No dimensional accuracy interaction effect was found between dimension location and SLS build position.

While the fabricated PD-AFOs demonstrated acceptable dimensional accuracy and fit customization, several possible sources of error exist that could affect the dimensional accuracy of the PD-AFO. One source could be part shrinkage due to features, such as geometry, that could not be accounted for in the scale part [24]. Furthermore, the FaroArm is reported to have $\pm 0.036 \mathrm{~mm}$ accuracy [28]; however, human error in using the FaroArm while measuring the PD-AFO dimensions could alter the dimensional accuracy results. Additionally, possible sources of error exist throughout the subject fit characterization, virtual orthopedic alignment, and CAD model customization stages that could influence the PD-AFO fit customization results related to the actual shape of the subject's limb. These sources may include error in digitizing the landmarks due to subject movement or inaccuracy by the researcher.

This study was limited in several ways. First, the effect of oblique build orientations or positions on the PD-AFO's dimensional accuracy was not tested. Also, the dimensional accuracy results are based on half-scale PD-AFOs, which were built because of build volume size constraints, yet the fit customization was assessed with the full-scale PD-AFOs. Dimensional accuracy may differ in full-scale PD-AFOs but, because of cost and complexity, measuring all PD-AFO sizes for this study was not feasible. Additionally, while PD-AFO comfort was assessed for nondisabled individuals, it was not evaluated for subjects with pathologies. Future studies will investigate the comfort of PD-AFOs customized and fabricated via this method for subjects with a range of impairments and functional limitations. Finally, while DuraForm EX is considered a durable material, the short- and long-term strength of PD-AFOs fabricated via this method have not been examined.

The fully parameterized nature of the PD-AFO CAD model lends itself extremely well to further customization. By a series of parameterized cardan rotations, the fully parameterized CAD model can readily be aligned into a full range of clinically indicated positions with precisely prescribed degrees of dorsiflexion/plantar flexion, inversion/eversion, and abduction/adduction of the shank relative to the foot. For instance, in the case of a plantar flexor contracture, the limb can be digitized in the contracted position, but the PD-AFO CAD model can be customized and subsequently fabricated with a prescribed amount of dorsiflexion to bring the patient's ankle into a more functional position [7]. Furthermore, the foot plate size and shape can be modified so the PD-AFO can be customized to a specific shoe or implemented without a shoe.

Cost analyses for rapid prototyping technologies must also be considered and are sensitive to numerous factors. Freeman and Wontorcik reported an hour-based cost range of US\$1,560 to US\$3,480 (\$60/h), which included preparation, material, and build time to fabricate a prosthetic test socket using stereolithography [29]. New (virgin) DuraForm EX Natural Plastic material costs approximately US\$4 per cubic inch. In this report, the PD-AFO customized for the female subject required 37 in. $^{3}$ of material at a cost of US\$148 for materials alone. Approximately 1 hour was needed for obtaining digitized landmark data and 
customizing the fully parameterized PD-AFO CAD model. An additional hour of technician time was required for executing the SLS manufacturing process, which can require approximately 24 hours of machine time to complete. Once built, the PD-AFO required a strap added on the cuff and padding inserted on the interior surface of the cuff and superior surface of the foot plate-approximately $30 \mathrm{~min}$ utes of effort. In total, 2.5 person hours were required. This is substantially less than traditional PD-AFO manufacturing methods and delivery times [7]. While this project was not subjected to business-related costs associated with build time, machine depreciation, material waste, and annual maintenance costs, a quote of US\$3,327.84 obtained from a commercial SLS manufacturing site to manufacture one full-scale PD-AFO indicated that a substantial level of overhead may accompany current commercial manufacturing models. As a comparison, one patient reported a 2007 cost for a manually fabricated PD-AFO of US\$8,000 per orthosis.

The fit customization and manufacturing framework described is one component of the entire automated customization and manufacturing framework, which incorporates customization of PD-AFO functional characteristics, including select foot plate characteristics and PD-AFO bending stiffness. While tuning the PD-AFOs used in this study to 50 percent of the subjects' natural pseudo ankle stiffness was based on observations of commercial carbon-fiber AFOs that patients found comfortable, the stiffnesses of the PD-AFOs used in this study were higher than many orthoses currently on the market [30]. The optimal settings of the functional characteristics as well as their influence on gait kinetics and kinematics have yet to be determined. With the help of clinical expertise and further biomechanical investigation, the CAD model parameter settings controlling the functional characteristics could be prescribed to optimally customize the PD-AFO to meet the unique needs of every patient. Furthermore, the PD-AFO customization and manufacturing framework prescribes use of CAD-compatible finite element analysis and optimization tools to analytically tune functional characteristics such as strut and foot plate bending stiffnesses to automatically obtain results similar to those manually achieved by Faustini et al. [5]. The fully parameterized nature of the PDAFO CAD model supports the optimization of parameters to achieve a desired PD-AFO functional characteristic. Thus, customized stiffness characteristics can be rapidly obtained by automatically converging on PD-AFO parameter settings related to bending stiffness. After fabrication and dimensional accuracy assessment, the stiffness characteristics can also be assessed through experimental testing. Lastly, as a supplement to the design process, the PD-AFO function customization may be evaluated while the subject walks with the PD-AFO through motion analysis and other experimental techniques.

\section{CONCLUSIONS}

Dimensional accuracy of the rapid fit customization and PD-AFO manufacturing framework detailed in this article is well within tolerances suggested in the literature. While the material costs appear reasonable, the time savings over traditional PD-AFO fabrication methods appear substantial. The potential for objective and repeatable customization of biomechanically designed orthoses supports the field's transformation from a craft-based industry to a modern clinical specialty. Mass exploitation of the automated customization and manufacturing framework would require a centralized manufacturing system that leverages the efficiencies and minimizes the overhead and businessrelated expenses of an SLS manufacturing paradigm.

\section{ACKNOWLEDGMENTS}

\section{Author Contributions:}

Study concept and design: S. J. Stanhope, E. S. Schrank. Acquisition of data: E. S. Schrank.

Analysis and interpretation of data: E. S. Schrank, S. J. Stanhope. Drafting of manuscript: E. S. Schrank, S. J. Stanhope.

Critical revision of manuscript for important intellectual content: S. J. Stanhope, E. S. Schrank.

Statistical analysis: E. S. Schrank.

Obtained funding: S. J. Stanhope, E. S. Schrank.

Administrative, technical, or material support: S. J. Stanhope.

Study supervision: S. J. Stanhope.

Financial Disclosures: The authors have declared that no competing interests exist.

Funding/Support: This material was based on work supported in part by the University of Delaware, the National Science Foundation (Graduate Research Fellowship Program), and the intramural research program of the Eunice Kennedy Shriver National Institute of Child Health and Human Development. Manufacturing of the PD-AFOs was conducted under a cooperative research and development agreement between the University of Delaware and the U.S. Army Edgewood Chemical Biological Center in Aberdeen Proving Ground, Maryland.

Additional Contributions: We recognize the assistance of Mr. Kevin Wallace and Mr. Rick Moore.

Institutional Review: This study was approved by the institutional review boards of both the National Institute of Child Health and 
Human Development at the National Institutes of Health and the University of Delaware.

Participant Follow-Up: Study subjects have been referred to our Web site's publication section for publications related to this research study.

\section{REFERENCES}

1. Brault MW. Americans with disabilities: 2005. Washington (DC): U.S. Census Bureau; 2008. p. 70-117.

2. Kepple TM, Siegel KL, Stanhope SJ. Relative contributions of the lower extremity joint moments to forward progression and support during gait. Gait Posture. 1997;6(1):1-8. DOI:10.1016/S0966-6362(96)01094-6

3. Neptune RR, Kautz SA, Zajac FE. Contributions of the individual ankle plantar flexors to support forward progression and swing initiation during walking. J Biomech. 2001; 34(11):1387-98. [PMID: 11672713]

DOI:10.1016/S0021-9290(01)00105-1

4. Pomeranz B, Adler U, Shenoy N, Macaluso C, Parikh S. Prosthetics and orthotics for the older adult with a physical disability. Clin Geriatr Med. 2006;22(2):377-94.

[PMID: 16627084]

DOI:10.1016/j.cger.2005.12.006

5. Faustini MC, Neptune RR, Crawford RH, Stanhope SJ. Manufacture of passive dynamic ankle-foot orthoses using selective laser sintering. IEEE Trans Biomed Eng. 2008; 55(2 Pt 1):784-90. [PMID: 18270017] DOI:10.1109/TBME.2007.912638

6. Loke M. Dynamic Bracing Solutions [Internet]. San Diego (CA): Dynamic Bracing Solutions; 2010 [updated 2010 Oct 8; cited 2010 Apr 27]. Available from: http://www.dynamicbracingsolutions.net/

7. Arizona AFO [Internet]. Mesa (AZ): Arizona AFO; 2010 [cited 2010 Apr 27]. Available from: http://www.arizonaafo.com/

8. Bartonek A, Eriksson M, Gutierrez-Farewik EM. A new carbon fibre spring orthosis for children with plantarflexor weakness. Gait Posture. 2007;25(4):652-56. [PMID: 16962328] DOI:10.1016/j.gaitpost.2006.07.013

9. Desloovere K, Monlenaera G, Van Gestel L, Huenaerts C, Van Campenhout A, Callewaert B, Van de Walle P, Seyler J. How can push-off be preserved during use of an ankle foot orthosis in children with hemiplegia? A prospective controlled study. Gait Posture. 2006;24(2):142-51.

[PMID: 16934470]

DOI:10.1016/j.gaitpost.2006.08.003

10. Bedotto RA. Biomechanical assessment and treatment in lower extremity prosthetics and orthotics: A clinical perspective. Phys Med Rehabil Clin N Am. 2006;17(1):203-43.
[PMID: 16517352]

DOI:10.1016/j.pmr.2005.10.007

11. Nair PM, Rooney KL, Kautz SA, Behrman AL. Stepping with an ankle foot orthosis re-examined: A mechanical perspective for clinical decision making. Clin Biomech (Bristol, Avon). 2010;25(6):618-22. [PMID: 20362373] DOI:10.1016/j.clinbiomech.2010.03.001

12. Convery P, Greig RJ, Ross RS, Sockalingam S. A three centre study of the variability of ankle foot orthoses due to fabrication and grade of polypropylene. Prosthet Orthot Int. 2004;28(2):175-82. [PMID: 15382811]

DOI:10.1080/03093640408726702

13. Nelson KM, Kepple TM, Siegel KL, Halstead LS, Stanhope SJ. Ankle foot orthosis contribution to net ankle moments in gait. Proceedings of the 27th Annual Meeting of the American Society of Biomechanics; 2003 Sep 25-27; Toledo, OH.

14. AOPA Fact Sheet [Internet]. Alexandria (VA): American Orthotic \& Prosthetic Association; 2009 [cited 2009 Oct 5]. Available from:

http://www.aopanet.org/index.php?option=com_content\&view =article\&id=76\&Itemid $=94$

15. Condie DN. The modern era of orthotics. Prosthet Orthot Int. 2008;32(3):313-23. [PMID: 18825575] DOI:10.1080/03093640802113006

16. Doxey GE. Clinical use and fabrication of molded thermoplastic foot orthotic devices. Suggestions from the field. Phys Ther. 1985;65(11):1679-82. [PMID: 4059331]

17. Darling AL, Sun W. Orthotic design through 3D reconstruction: A passive-assistance ankle-foot orthotic. Appl Bion Biomech. 2006;3(2):93-100. DOI:10.1533/abbi.2005.0014

18. Rogers B, Bosker GW, Crawford RH, Faustini MC, Neptune RR, Walden G, Gitter AJ. Advanced trans-tibial socket fabrication using selective laser sintering. Prosthet Orthot Int. 2007;31(1):88-100. [PMID: 17365888]

DOI:10.1080/03093640600983923

19. Faustini MC, Neptune RR, Crawford RH, Rogers WE, Bosker G. An experimental and theoretical framework for manufacturing prosthetic sockets for transtibial amputees. IEEE Trans Neural Syst Rehabil Eng. 2006;14(3):304-10. [PMID: 17009490]

DOI:10.1109/TNSRE.2006.881570

20. South BJ, Fey NP, Bosker G, Neptune RR. Manufacture of energy storage and return prosthetic feet using selective laser sintering. J Biomech Eng. 2010;132(1):015001.

[PMID: 20524754]

DOI:10.1115/1.4000166

21. Pallari JH, Dalgarno KW, Woodburn J. Mass customization of foot orthoses for rheumatoid arthritis using selective laser sintering. IEEE Trans Biomed Eng. 2010;57(7):1750-56.

[PMID: 20211798]

DOI:10.1109/TBME.2010.2044178 
22. Berry E, Brown JM, Connell M, Craven CM, Efford ND, Radjenovic A, Smith MA. Preliminary experience with medical applications of rapid prototyping by selective laser sintering. Med Eng Phys. 1997;19(1):90-96. [PMID: 9140877] DOI:10.1016/S1350-4533(96)00039-2

23. Ibrahim D, Broilo TL, Heitz C, De Oliveira MG, De Oliveira HW, Nobre SM, Dos Santos Filho JH, Silva DN. Dimensional error of selective laser sintering, three-dimensional printing and PolyJet models in the reproduction of mandibular anatomy. J Craniomaxillofac Surg. 2009;37(3):167-73. [PMID: 19056288] DOI:10.1016/j.jcms.2008.10.008

24. Senthilkumaran K, Pandey PM, Rao PV. Influence of building strategies on the accuracy of parts in selective laser sintering. Mater Des. 2009;30(8):2946-54.

25. Silva DN, Gerhardt de Oliveira MG, Meurer E, Meurer MI, Lopes da Silva JV, Santa-Bárbara A. Dimensional error in selective laser sintering and 3D-printing of models for craniomaxillary anatomy reconstruction. J Craniomaxillofac Surg. 2008;36(8):443-49. [PMID: 18579391]

DOI:10.1016/j.jcms.2008.04.003

26. Razzook AR, Nelson KM, Siegel KL, Stanhope SJ. Can passive dynamic ankle foot orthoses replicate natural ankle stiffness? Proceedings of the 28th Annual Meeting of the American Society of Biomechanics; 2005 Jul 31-Aug 5; Cleveland, $\mathrm{OH}$.

27. Ng P, Lee PS, Goh JC. Prosthetic sockets fabrication using rapid prototyping technology. Rapid Prototyp J. 2002;8(1): 53-59. DOI:10.1108/13552540210413310
28. FaroArm - Complete wireless freedom [Internet]. Lake Mary (FL): FARO Technologies; 2010 [cited 2009 Oct 6]. Available from: http://measuring-arms.faro.com/distri/features/

29. Freeman D, Wontorcik L. Stereolithography and prosthetic test socket manufacture: A cost/benefit analysis. J Prosthet Orthot. 1998;10(1):17-20. DOI:10.1097/00008526-199801010-00005

30. Ringleb SI, Armstrong T, Berglund LJ, Kitaoka HB, Kaufman KR. Stiffness of the Arizona ankle-foot orthosis before and after modification for gait analysis. J Prosthet Orthot 2009;21(4):204-7. DOI:10.1097/JPO.0b013e3181bfb28e

Submitted for publication December 10, 2009. Accepted in revised form September 8, 2010.

This article and any supplementary material should be cited as follows:

Schrank ES, Stanhope SJ. Dimensional accuracy of ankle-foot orthoses constructed by rapid customization and manufacturing framework. J Rehabil Res Dev. 2011; 48(1):31-42.

DOI:10.1682/JRRD.2009.12.0195

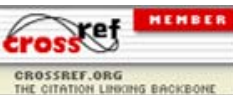

